\title{
Theta Oscillations Organize Spiking Activity in Higher- Order Visual Thalamus during Sustained Attention
}

\author{
(DChunxiu Yu, ${ }^{1}$ Yuhui Li, ${ }^{1}$ lain M. Stitt, ${ }^{1}$ Zhe Charles Zhou, ${ }^{1,5}$ CKristin K. Sellers, ${ }^{1}$ and ${ }^{1}$ Flavio \\ Frohlich ${ }^{1,2,3,4,5}$
}

\section{DOI:http://dx.doi.org/10.1523/ENEURO.0384-17.2018}

${ }^{1}$ Department of Psychiatry, University of North Carolina at Chapel Hill, Chapel Hill, NC 27599, ${ }^{2}$ Department of Neurology, University of North Carolina at Chapel Hill, Chapel Hill, NC 27599, ${ }^{3}$ Department of Biomedical Engineering, University of North Carolina at Chapel Hill, Chapel Hill, NC 27599, ${ }^{4}$ Department of Cell Biology and Physiology, University of North Carolina at Chapel Hill, Chapel Hill, NC 27599, and ${ }^{5}$ Neuroscience Center, University of North Carolina at Chapel Hill, Chapel Hill, NC 27599

\begin{abstract}
Higher-order visual thalamus plays a fundamental but poorly understood role in attention-demanding tasks. To investigate how neuronal dynamics in higher-order visual thalamus are modulated by sustained attention, we performed multichannel electrophysiological recordings in the lateral posterior-pulvinar complex (LP/pulvinar) in the ferret (Mustela putorius furo). We recorded single unit activity and local field potential (LFP) during the performance of the five-choice serial reaction time task (5-CSRTT), which is used in both humans and animals as an assay of sustained attention. We found that half of the units exhibited an increasing firing rate during the delay period before stimulus onset (attention-modulated units). In contrast, the non-attention-modulated units responded to the stimulus, but not during the delay period. Spike-field coherence (SFC) of only the attentionmodulated neurons significantly increased from the start of the delay period until screen touch, predominantly in the $\theta$ frequency band. In addition, $\theta$ power and $\theta / \gamma$ phase amplitude coupling (PAC) were elevated throughout the delay period. Our findings suggest that the $\theta$ oscillation plays a central role in orchestrating thalamic signaling during sustained attention.
\end{abstract}

Key words: 5-CSRTT; ferret; oscillations; sustained attention; thalamus; $\theta-\gamma$ coupling

\section{Significance Statement}

Impaired sustained attention can be deadly, as illustrated by the number of motor vehicle accidents that are caused by drivers not reacting quickly enough to unexpected events on the road. Understanding how electrical signaling in higher-order visual nuclei, such as the LP/pulvinar, is modulated during tasks that require sustained attention is an important step in achieving a mechanistic understanding of sustained attention, which will eventually lead to new strategies to prevent and treat impairment in sustained attention.

\section{Introduction}

Sustained attention is defined as the allocation of processing resources to rare but important events during prolonged periods of time (Sarter et al., 2001). Sustained attention is a key element of attention models (Mirsky et al., 1991) and integrates multiple behavioral processes including executive control for controlling competing impulses and executing planned actions to appropriate stimuli (Cohen et al., 1993).

In humans, sustained attention is typically measured with the continuous performance task (CPT), which requires the participant to respond to an infrequent stimulus 
over prolonged time. Neuroimaging studies support an active role of the fronto-parietal attention network in sustained attention (Lewin et al., 1996) but subcortical structures have also been proposed to be part of the network substrate of sustained attention (Riccio et al., 2002). Sustained attention is not only impaired in attention deficit hyperactivity disorder (ADHD; Barkley, 1997, Bellgrove et al., 2006, Johnson et al., 2007) but also depression (Cohen et al., 2001), bipolar disorder (Martínez-Arán et al., 2000), and schizophrenia (Liu et al., 2002). Interestingly, impairment of sustained attention persists in patients with bipolar disorder (Clark et al., 2002) and major depressive disorder (Paelecke-Habermann et al., 2005) even after achieving remission.

In animal model species, one task that has been extensively used to delineate the substrate of sustained attention is the five-choice serial reaction time task (5-CSRTT) that probes spatial sustained attention (Bari et al., 2008). In the 5-CSRTT, animals respond to a visual stimulus at one of five stimulus locations after a delay period. The 5 -CSRTT is a widely used task that has provided fundamental insights into the cellular and molecular mechanisms of sustained attention, and is used for the evaluation of candidate compounds for the treatment of ADHD (Day et al., 2007).

Despite the extensive study of sustained attention at the behavioral level in both humans and animal models, surprisingly little is known about the circuit dynamics of sustained attention. Recently, we reported that the 5-CSRTT engaged synchrony of oscillatory activity in the fronto-parietal network of the ferret (Sellers et al., 2016; Zhou et al., 2016b). Phase synchronization both at the microscale of neuronal action potentials and the mesoscale of the population synaptic activity reflected in the local field potential (LFP) was elevated in the $\theta$ frequency range.

In contrast, the role of subcortical structures in oscillatory network interactions during sustained attention has remained mostly unknown. The thalamus plays a major role in regulating the thalamo-cortical network dynamics that reflect vigilance levels (Llinás and Steriade, 2006; Stitt et al., 2017). Since vigilance requires sustained attention, we asked if higher-order thalamus is engaged in the 5-CSRTT. In particular, we investigated how spiking and oscillatory network activity is modulated during the delay period during which the animals pay attention to the potential stimulus locations.

K.K.S. performed research; C.Y., Y.L., and I.M.S. analyzed data; C.Y., Y.L., I.M.S., and F.F. wrote the paper.

This work was partially supported by the National Institute of Mental Health Award R01MH101547 (to F.F.).

Correspondence should be addressed to Flavio Frhlich, 115 Mason Farm Road, NRB 4109F, Chapel Hill, NC 27599, E-mail: flavio_frohlich@med.unc.edu. DOI:http://dx.doi.org/10.1523/ENEURO.0384-17.2018

Copyright @ 2018 Yu et al.

This is an open-access article distributed under the terms of the Creative Commons Attribution 4.0 International license, which permits unrestricted use, distribution and reproduction in any medium provided that the original work is properly attributed.

\section{Materials and Methods}

Three spayed female ferrets (Mustela putorius furo, 4 months of age at the beginning of the experiments) were trained to reach satisfactory task performance and subsequently implanted with electrode arrays in the LP/ pulvinar complex for combining neurophysiological measurements with behavioral data collection. All procedures were approved by the Institutional Animal Care and Use Committee of the University of North Carolina at Chapel Hill and followed National Institutes of Health guidelines for the care and use of laboratory animals.

\section{Behavioral apparatus}

Ferrets were trained in a touchscreen-based version of the 5-Choice Serial Reaction Time Task (5-CSRTT; Bari et al., 2008). Training took place in an enclosed and sound-attenuated custom-built operant chamber $(51 \times$ $\left.61 \times 61 \mathrm{~cm}^{3}\right)$. The chamber consisted of a touchscreen monitor (Acer T232HL 23-inch touchscreen LCD display) covered with a black Plexiglas mask. The mask exhibited five equally sized square cut-outs (windows, $7 \times 7 \mathrm{~cm}^{2}$ ), in one of which the stimulus was presented in each trial. Below the openings, there was a shelf on which the animals could comfortably rest their front legs when nosepoking the touchscreen. The opposite wall was equipped with a lick spout centrally positioned $6 \mathrm{~cm}$ above the floor for water delivery combined with an infrared (IR)-based proximity detector and an LED light. The behavioral chamber also included a "house light" mounted on the ceiling of the chamber opposite the monitor to provide feedback for correct and incorrect trials and a speaker (HP Compact 2.0 speaker) to deliver auditory tones. IR videography was performed during all sessions (Microsoft LifeCam Cinema 720p HD Webcam with filter that blocks IR light removed). The entire behavioral setup was controlled by a data acquisition device (USB 6212, National Instruments) and custom-written Matlab scripts (Mathworks) that used functionality from the Psychophysics toolbox (Brainard, 1997) for precise temporal control of stimulus presentation.

\section{5-CSRTT}

In the 5-CSRTT (Bari et al., 2008), the animals initiated a trial by approaching the lick-spout at the rear end of the behavior chamber. On initiation, a 5-s delay period started during which no stimuli were presented and the ferrets had to prepare and sustain attention to the five windows (cut-outs in the mask on the touchscreen). After the delay ended, a white solid square (stimulus) was randomly presented for $2 \mathrm{~s}$ in one of five windows. For correct trials, touching the stimulus window during the $2 \mathrm{~s}$ of stimulus presentation or in the first $2 \mathrm{~s}$ after the stimulus was turned off (hold period, HP) triggered a 0.5-s tone, illumination of the lick spout light, and a water reward release $(0.2 \mathrm{ml})$ at the lick spout (Fig.1A, left). The lick spout light was on until the animal nose poked into the lick spout. For incorrect trials, touching a window before the stimulus onset (PreTouch) or touching an adjacent incorrect window after 5-s delay (MissTouch), or failing to respond to 


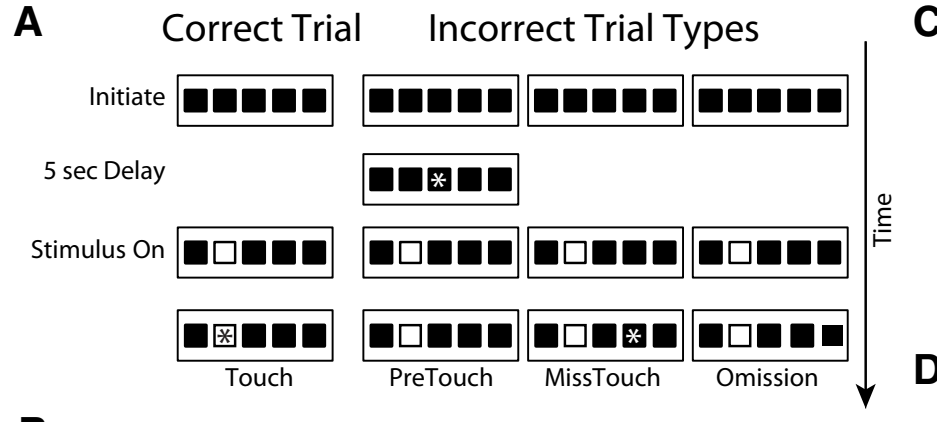

B
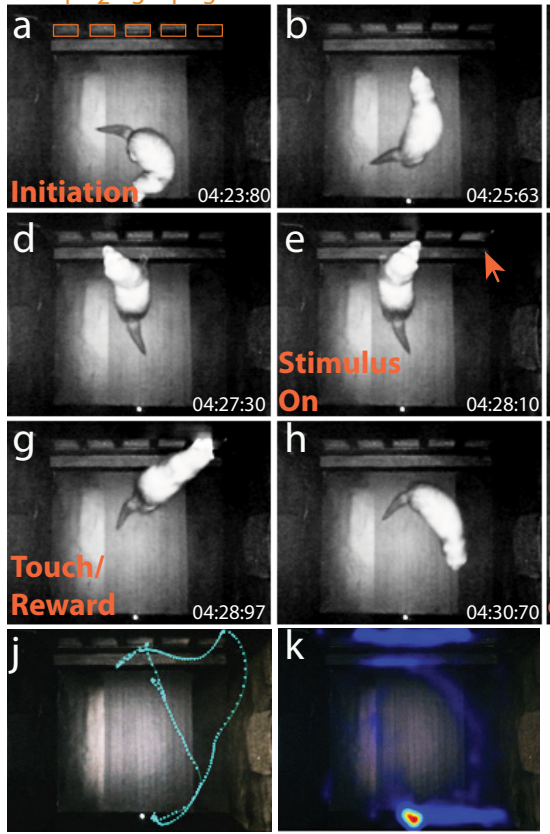
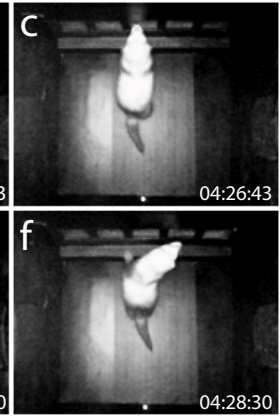

C
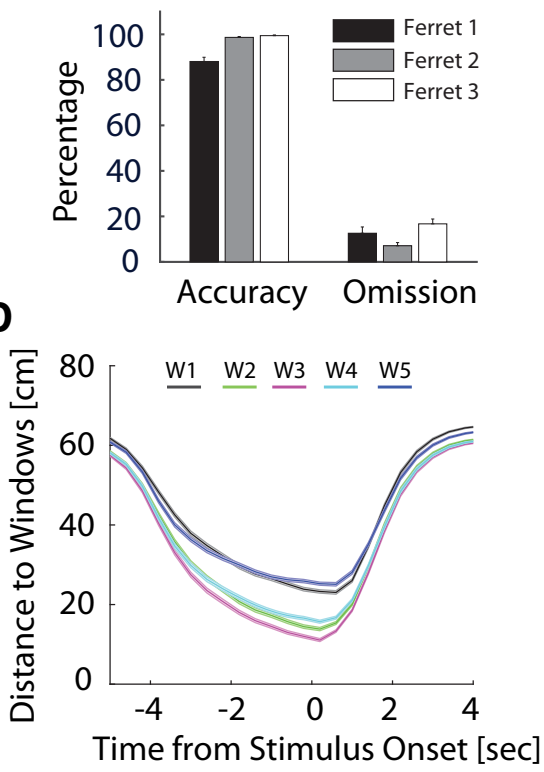

E
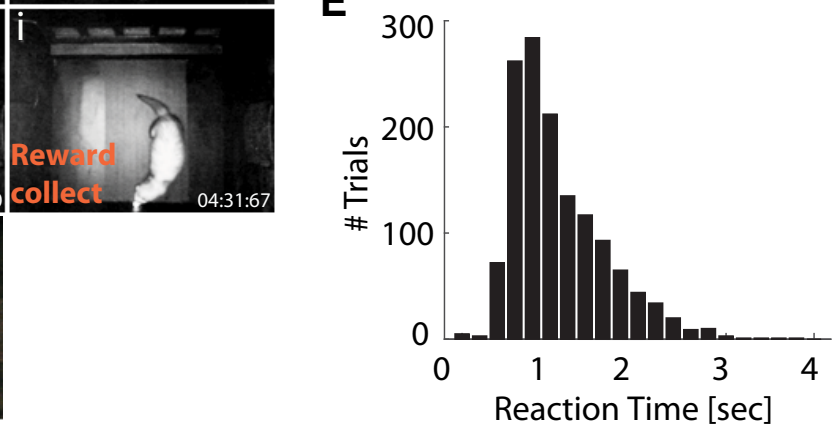

Figure 1. Sustained attention task in freely-moving ferrets. A, Illustration of trial sequences of the 5-CSRTT. Each trial begins with illumination of the water spout, which is centrally placed on the back wall of the chamber. The ferret initiates the trial by approaching the water spout, which is equipped with an IR proximity sensor. Then, the spout light is extinguished and the 5-s delay period starts during which the animal is required to sustain attention to the five windows on the front wall of the chamber. A white solid square (stimulus) will randomly present in one of five windows after the delay ends. Nose-poke to the stimulus window during stimulus presentation (2 s) or in the first $2 \mathrm{~s}$ after stimulus offset (HP) triggers a tone and delivery of water reward at the spout (correct trial, left). Touch by the ferret is indicated with an asterisk. If the ferret responds before the stimulus (PreTouch), or touches an incorrect window (MissTouch), or fails to respond before the end of the HP (omission), a 6-s time-out (TO) period is introduced where the house light is on and no water is delivered (right). After collecting the reward (8-s time window) or at the end of the TO period, a new trial can be started. $\boldsymbol{B}$, Representative photographs of one animal during a single trial: $a$, initiation; $b$, turn to the stimulus windows (numbered 1 through 5 in subpanel $a$ ); $c-d$, paws on the platform and checking for occurrence of stimulus; $e$, stimulus on; $f$, find the stimulus; $g$, nose poke the stimulus window; $h$, turn back to collect the reward; $i$, collect the reward; $j$, complete trajectory for a single trial obtained from video tracking; $k$, heatmap of animal locations during the session. Time stamps are shown in the corner of each frame. $\boldsymbol{C}$, Behavioral performance. Mean accuracy and omission rates across sessions. Error bars: SEM across sessions. $\boldsymbol{D}$, Mean distance between animal location and stimulus location as a function of time for correct trials. The shorter distances to middle windows (W2 - W4) indicate that animals were centered relative to the stimulus windows before stimulus onset. $\boldsymbol{E}$, Distribution of touch reaction times $(\mathrm{RT})$ for correct trials. In most trials, the correct window was touched during stimulus presentation (RT $<2 \mathrm{~s}$ ).

the stimulus at all (NoTouch, omission) caused a $1 \mathrm{~s}$ white noise auditory stimulus, illumination of the house light, and a subsequent 6-s time-out period, in which no water was delivered (Fig. $1 A$, right). Following the $8 \mathrm{~s}$ inter-trial interval after correct responses or 6-s time-out period after incorrect responses, the lick-spout light turned on to indicate the availability of the next trial. The session was terminated after 60 trials or $40 \mathrm{~min}$, whichever came first. Criteria of $>80 \%$ accurate stimulus detection and $<20 \%$ omission for at least five consecutive sessions were used during the training.

\section{Behavioral training}

Ferrets were water deprived and received free access to water $(60 \mathrm{ml})$ each day after training and testing. Body weight was monitored daily and never dropped below $85 \%$ which would have triggered discontinuation of water deprivation by protocol. The ferrets underwent a multistep 
training plan that included five subsequent training levels: (1) arena habituation; (2) touch-reward association; (3) single stimulus-reward association; (4) task initiation; and (5) 5-CSRTT. Following this training schedule, the ferrets were successively advanced to the final task, in which the ferrets were required to initiate the trial by nose-poking the water spout. Ferrets were trained and tested daily on a $5-d$ on/2-d off schedule.

\section{Surgery}

After successful training, the ferrets were surgically implanted with a chronic multichannel electrode array $(2 \times 8$ tungsten electrode array, 250- $\mu \mathrm{m}$ spacing, low-impedance reference electrode with the same length on the same array, MicroProbes Inc) in LP/pulvinar. Surgical procedure was similar to those previously described (Sellers et al., 2016; Zhou et al., 2016c). Briefly, ferrets were first injected with ketamine/xylazine $(30 \mathrm{mg} / \mathrm{kg}$ of ketamine, $1-2 \mathrm{mg} / \mathrm{kg}$ of xylazine, i.m.) for anesthesia induction, and then were intubated and anesthetized with inhaled isoflurane (1.5-2\%) in $100 \%$ medical oxygen (mechanically ventilated, $10-$ to $11-\mathrm{ml}$ volume, $50 \mathrm{bpm}$ ). Body temperature was maintained between $38-39^{\circ} \mathrm{C}$ with a water heating blanket. Electrocardiogram, end-tidal $\mathrm{CO}_{2}$ and partial oxygen saturation were monitored throughout surgery. The skull was exposed and a craniotomy was performed over the target area (centered at $13 \mathrm{~mm}$ anterior to lambda, $3.6 \mathrm{~mm}$ lateral from midline; $\mathrm{Yu}$ et al., 2016). A small slit was made into the dura before insertion of electrode array (7.4 mm from cortical surface). Electrode arrays were secured in place with dental cement and several non-penetrating skull screws. A separate ground wire was implanted in cortex of the same hemisphere. The wound margins were sutured together and anesthesia was reversed. Ferrets received standard postoperative care with $3 \mathrm{~d}$ of meloxicam for pain relief $(0.2 \mathrm{mg} / \mathrm{kg}$, i.m.) and $7 \mathrm{~d}$ of clavamox to prevent infection (12.5-13 $\mathrm{mg} / \mathrm{kg}$, oral).

\section{Electrophysiological recording}

The animals were given two weeks of recovery time before they were retrained on the final task to again reach stable performance (typically a few sessions). At this point, wireless electrophysiological recordings were performed with a MCS 2100 system (Multichannel Systems). Broadband data ( $1 \mathrm{~Hz}$ to $5 \mathrm{kHz}$ ) were collected (sampling rate: $20 \mathrm{kHz}$ ) and digitally stored for offline analysis. Simultaneously with acquisition of the neuronal data, timelocked acquisition of relevant behavioral events (trial initiation, screen touch, reward retrieval) was performed through digital input channels on the wireless recording system. Behavioral data were stored separately by a custom-written Matlab script. In addition, high-resolution IR videography (30 frames/s) was performed that was synchronized to the neuronal data acquisition.

\section{Histology}

To verify electrode locations in the LP/pulvinar complex, electrolytic lesions were induced at the completion of recording by passing current through the four electrodes (two middle and two corner) of the microelectrode array $(5 \mu \mathrm{A}, 10 \mathrm{~s}$, unipolar). The damage visible in the sections stem from this lesioning protocol. Animals were then humanely euthanized with an overdose of sodium pentobarbital (intravenous injection) and perfused with 0.1 M PBS and $4 \%$ paraformaldehyde solution in $0.1 \mathrm{M} \mathrm{PBS}$. The brains were post-fixed and cut in $60-\mu \mathrm{m}$ coronal sections using a cryostat (CM3050S, Leica Microsystems), and then stained for cytochrome oxidase (Yu et al., 2016). Imaging was acquired using a widefield microscope (Nikon Eclipse 80i, Nikon Instruments). Electrodes outside of LP/pulvinar were excluded from analysis.

\section{Data analysis}

Behavioral data were analyzed with custom-written Matlab scripts. The main measurements of interest were: (1) identity of the window touched, (2) reaction time after visual stimulus onset, and (3) animal location relative to windows on touchscreen. Equally, MissTouch, NoTouch and PreTouch trials were also tracked. Performance accuracy in detecting the stimulus was defined as percentage of the number of correct responses divided by the total number of correct and incorrect (MissTouch) responses. The video recordings were analyzed offline with Ethovision XT (Noldus) and the three-point animaltracking module to detect the animal location (sample rate, 30 frames/s). Heatmaps were generated for demonstration of animal trajectories. We also manually reviewed video recordings and coded the orientation of the animal immediately before the stimulus onset. Only correct trials with the animal orienting to the screen at the time of stimulus onset were included in further analysis.

Electrophysiology data were analyzed by a combination of custom-written Matlab scripts. Trials exhibiting simultaneous large amplitude deflections across channels (artifacts in electrophysiological recordings) were excluded. Single units were extract with the Plexon Offline Sorter (Plexon Inc) spike sorting software. Briefly, multiunit activity was detected by high-pass filtering the raw continuous data with a Butterworth 2nd order filter with $300-\mathrm{Hz}$ cutoff. Action potentials were detected by threshold crossing ( -4 times the SD of the high-pass filtered signal). Spikes were identified, collected and sorted using the T-distribution expectation maximization algorithm (Shoham et al., 2003) and manual inspection with the Plexon Offline Sorter. Spikes with shorter than 1-ms refractory period were removed. Neural activity within a window of $-7-5 \mathrm{~s}$ aligned on stimulus onset was averaged in 200-ms bins and averaged across trials to construct the peristimulus time histogram (PSTH). The PSTHs were then $z$-scored by subtracting the mean baseline firing rate (measured from a baseline window from -2 to $0 \mathrm{~s}$ before trial initiation) and dividing by the standard deviation. To classify "attention-modulated" versus non-attentionmodulated units, neural activity within $2 \mathrm{~s}$ before the stimulus onset was compared to a baseline window from -2 to $0 \mathrm{~s}$ before the trial initiation (two-tailed $t$ test, $p<$ 0.05 ). The 2-s window was chosen based on visual inspection of the behavioral video. The ferrets usually began facing the windows and remained in an oriented posture around $2 \mathrm{~s}$ before stimulus onset. 
To investigate the rhythmic structure of LP/pulvinar activity, LFPs were computed by low-pass filtering the raw continuous data with a Butterworth 2nd order filter with $300-\mathrm{Hz}$ cutoff. To study the synchrony between LP/ pulvinar spikes and the LFP at different epochs during the task, we computed the spike-field coherence (SFC) of simultaneously recorded spike trains and LFP using multitaper analysis provided by the Chronux data analysis toolbox (Saalmann et al., 2012). The SFC was calculated in $2 \mathrm{~s}$ windows using the coherencycpt function for each epoch (before initiation, before stimulus onset, before screen touch and after screen touch). We corrected coherence values to remove the effects of unequal number of trials in each session (Bokil et al., 2007; Saalmann et al., 2012). The corrected SFC was computed using the formula

$$
T(f)=\operatorname{atanh}(C(f))-1 /(d f-2),
$$

where $C(f)$ is the raw coherence value; $d f$ is the degrees of freedom; $\mathrm{df}=2 * \mathrm{~K} * \mathrm{~N}$, where $\mathrm{K}$ is the number of tapers and $\mathrm{N}$ is the number of trials. The corrected coherence estimates were pooled across the population. Two-way ANOVA was used to compare the modulation of the coherence at different epochs during the task. We calculated the spike-triggered average (STA) of the LFP by extracting segments of the LFP from -1.2 to $1.2 \mathrm{~s}$ relative to the spike times of individual single units. Subsequently, all these LFP segments were aligned and averaged to compute the STA of the LFP. As a control, we shuffled the spike trains across different trials and re-computed the STA of the LFP. This manipulation only disrupts the relationship between the spikes and the LFP but preserves the temporal structure of the spike train within individual trials.

Cross-frequency phase amplitude coupling (PAC) was computed to assess the degree with which highfrequency oscillations are temporally organized by the phase of low-frequency oscillations in the LP/pulvinar. PAC was defined as the phase locking value (PLV) between low-frequency signals and amplitude fluctuations of high-frequency signals occurring at the lower carrier frequency. First, an LFP signal $x(t)$ was convolved with a low-frequency Morlet wavelet with carrier frequency $f_{1}$

$$
X\left(t, f_{1}\right)=x(t)^{\star} w\left(t, f_{1}\right)
$$

where $*$ denotes the convolution operation. Then the analytic amplitude of the same signal $x(t)$ at a higher carrier frequency $f_{2}$ was convolved with a wavelet with carrier frequency $f_{1}$

$$
X\left(t, f_{2}\right)=\left|X(t)^{\star} w\left(t, f_{2}\right)\right|^{*} w\left(t, f_{1}\right)
$$

The instantaneous phase of each time series was then computed by taking the argument of the real and imaginary components of the time frequency estimates. Finally, PAC is defined as the PLV between low and highfrequency signal components

$$
\operatorname{PAC}\left(f_{1}, f_{2}\right)=\frac{1}{N}\left|\sum_{n=1}^{N} e^{i\left(\theta_{n}^{f_{1}}-\theta_{n}^{f_{2}}\right)}\right|
$$

PAC values were bound between 0 and 1 , with 0 indicating no relationship, and 1 perfect alignment of highfrequency amplitude modulations with low-frequency phase. To examine PAC extensively in frequencyfrequency parameter space, we computed PAC between low frequencies $\left(f_{1}\right)$ ranging from 1 to $10 \mathrm{~Hz}$ in $0.25-\mathrm{Hz}$ steps, and high frequencies $\left(f_{2}\right)$ from 10 to $80 \mathrm{~Hz}$ in $1-\mathrm{Hz}$ steps. This analysis revealed prominent PAC between $\theta$ and $\gamma$ frequencies in LP/pulvinar. After identifying this frequency-frequency band of interest, we repeated analysis using a phase preserving $4^{\text {th }}$ order Butterworth filter and Hilbert transform instead of Morlet wavelet convolution to maximally capture time frequency fluctuations in the $\theta / \gamma$ band of interest $(\theta, 3-6 \mathrm{~Hz} ; \gamma, 30-60 \mathrm{~Hz})$. Finally, to investigate how $\theta / \gamma$ PAC is modulated by the 5-CSRT, we computed PAC in sliding windows of $200 \mathrm{~ms}$ (step size $100 \mathrm{~ms}$ ) aligned to stimulus onset.

\section{Results}

\section{Behavioral performance}

To probe the role of LP/pulvinar neuronal dynamics in sustained attention, we trained three ferrets to perform a 5-CSRTT (Fig. 1A,B). The 5-CSRTT was designed as a task in which freely moving animals make a choice in each trial to get a reward. Trials were self-initiated at a lick spout in the back of the behavioral apparatus and a touchscreen with five stimulus locations was used to display the targets and capture the behavioral responses. All ferrets reached criterion performance across recording sessions with high accuracy (Fig. $1 C$; mean \pm SEM, ferret 1: $88.1 \% \pm 1.85 \%$, ferret $2: 98.7 \% \pm 0.38 \%$, ferret 3 : $99.4 \% \pm 0.29 \%$ ) and low omission rates (ferret 1: $12.6 \pm$ $2.77 \%$, ferret 2: $7.10 \% \pm 1.40 \%$, ferret $3: 16.7 \% \pm$ $2.12 \%)$. Video tracking data confirmed that animals moved toward the screen during the delay period (Fig. $1 D$ ), with animals reaching their final position close to the screen $\sim 2 s$ before stimulus onset. This implies that ferrets allocated attention to the stimulus windows in anticipation of the visual stimulus during this time period. In addition, the reaction time distribution for correct trials indicated that animals responded quickly to stimuli, with most responses within $2 \mathrm{~s}$ of stimulus onset (Fig. 1E). We used criteria of $>80 \%$ accurate stimulus detection and $<20 \%$ omission for at least five consecutive sessions to determine that an animal has learnt the task. Typically, 12 sessions were used before the surgery. After surgery, approximately one to three sessions of recovery training were needed before the behavioral performance reached the criteria again and the electrophysiological recording were started. We then asked if neuronal firing in the $\mathrm{LP} /$ pulvinar was modulated during the delay period.

\section{Single unit activity in LP/pulvinar}

We implanted microelectrode arrays into LP/Pulvinar and confirmed implantation locations through histologic reconstruction of the recording sites (Fig. $2 A$ ). We found 
A

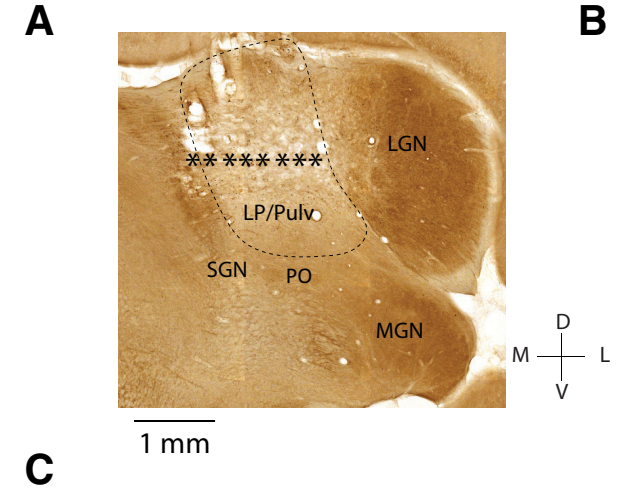

C

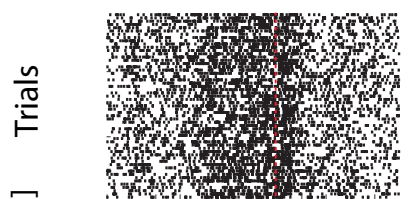

B

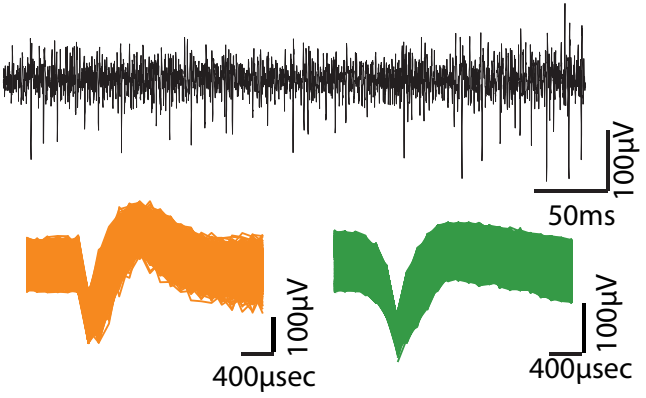

$\mathbf{E}$

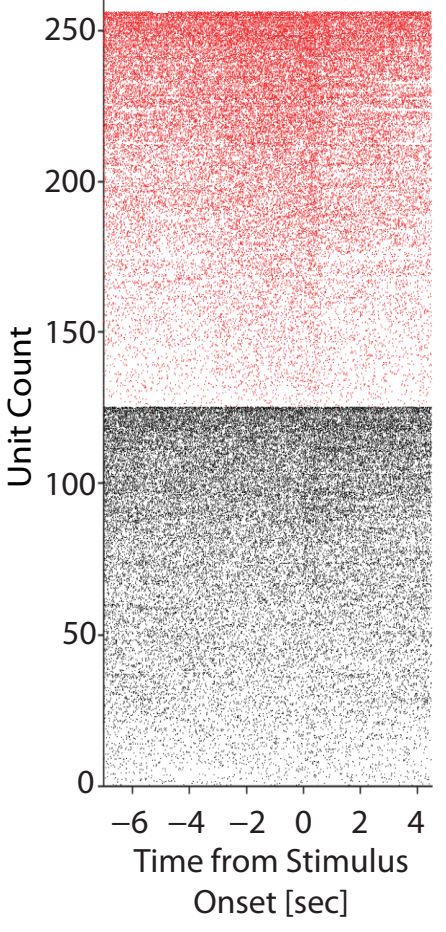

Figure 2. Single-unit responses during task performance. A, Representative coronal section; stars indicate the estimated locations of the electrode tips. Electrodes outside of LP/pulvinar (LP/Pulv) were excluded. LGN, lateral geniculate nucleus; MGN, medial geniculate nucleus; PO, nucleus of the posterior group; SGN, suprageniculate nucleus. $\boldsymbol{B}$, top, Example of high-pass filtered raw trace from representative recording session. Bottom, Action potentials of two representative single units. $\boldsymbol{C}$, Peri-event raster plots (top) and peri-event histograms of the corresponding firing rates (bottom) of two representative neurons. The unit on the left exhibited an increasing firing during the delay period, whereas the unit on the right did not show such modulation but rather increased its firing rate after stimulus onset. $\boldsymbol{D}, Z$-score normalized population firing rate of attention-modulated (red, $n=130$ ) and non-attention-modulated units (black, $n=129$ ) in correct trials (solid lines) and in incorrect trials (dotted lines). Attention-modulated units gradually increased their firing rate during the delay period. Both neuron types displayed a transient increase in firing rate after stimulus onset, with stronger responses in attention-modulated neurons. Shaded areas indicate SEM. E, Peri-event raster plots from all the attentionmodulated (red, $n=130$ ) and non-attention-modulated units (black, $n=129$ ) in all correct trials. Units from each category are sorted by their mean firing rate in the delay period.

that about half of the LP/pulvinar single neurons ( $n=$ $130 / 259$ units) displayed a progressively increasing firing rate during the delay period of the task (Fig. $2 B-E)$. Since no stimuli were present in any of the windows during the delay period, such elevated firing rates suggest that the excitability of this subpopulation of LP/pulvinar neurons is modulated by behavioral or mental engagement of the animal with the possible locations of the future stimulus (referred to as attention-modulated). The remaining 50\% of neurons ( $n=129 / 259)$ did not exhibit significant changes in firing rate during the delay period. However, both attention-modulated and non-attention-modulated LP/pulvinar neurons displayed increases in firing rate in response to the visual stimulus. This increase was significantly stronger for attention-modulated neurons (unpaired $t$ test, $\left.t_{(257)}=10.73, p<0.001\right)$. Furthermore, the attention-modulated neurons had higher firing rate in the correct trials compared to in the incorrect trials (Fig. 2D, solid red line compared to dotted red line, paired $t$ test, $\left.t_{(129)}=3.23, p=0.0016\right)$. Thus, the delay period of the 5 -CSRTT led to a ramping up of spiking activity in a subpopulation of LP/pulvinar neurons which were correlated to the behavioral performance.

\section{Modulation of SFC in the $\theta$ band}

We next asked whether the task-related activity of the neurons in LP/pulvinar exhibited a mesoscale organization that would be reflected in the LFP. To answer this question, we computed SFC of both attention-modulated and non-modulated neuron subpopulations during different epochs of trials. We found SFC in the $\theta$ band $(\sim 5 \mathrm{~Hz})$ throughout task performance (Fig. 3). We further confirmed the importance of the relationship between thalamic spikes and the $\theta$ oscillation by showing that the STA of the LFP exhibits rhythmic structure in the $\theta$ band (Fig. $3 B$, inset). Strikingly, SFC in the $\theta$ band differed for attention-modulated and non-modulated neurons. A twoway ANOVA with neuronal response type (attentionmodulated or non-modulated) and task segments (before initiation, before stimulus onset, before touch and after 
A

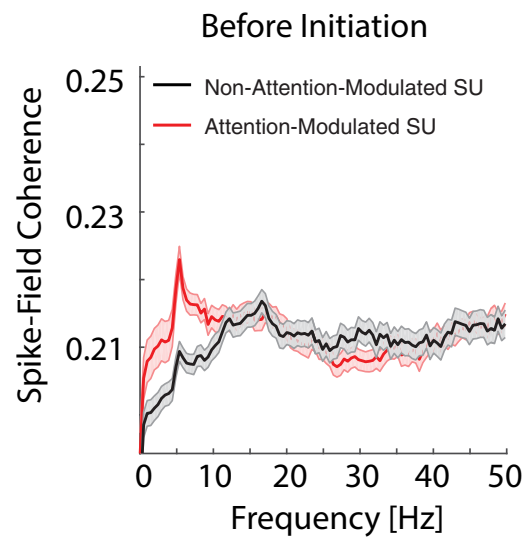

D

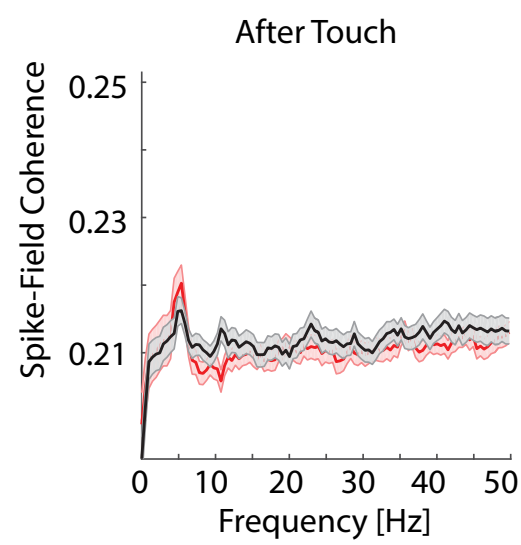

B

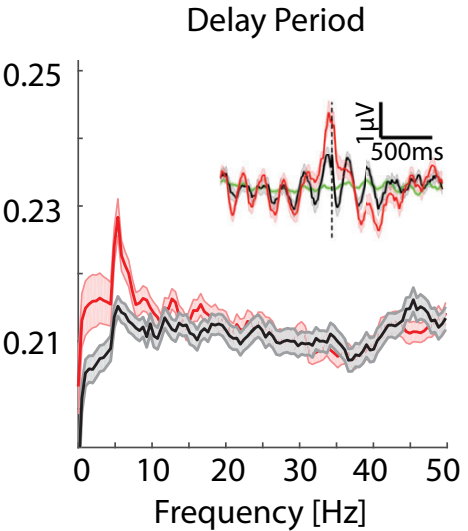

E
C

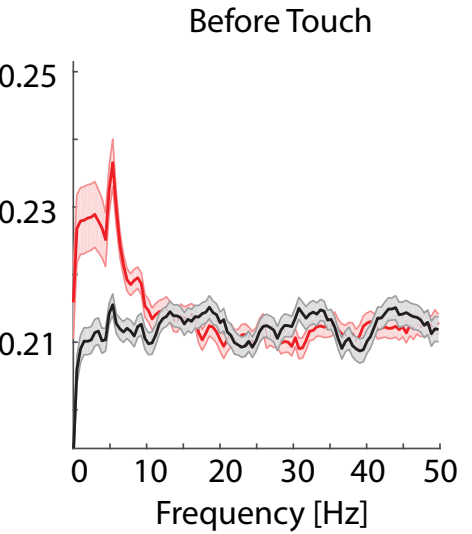

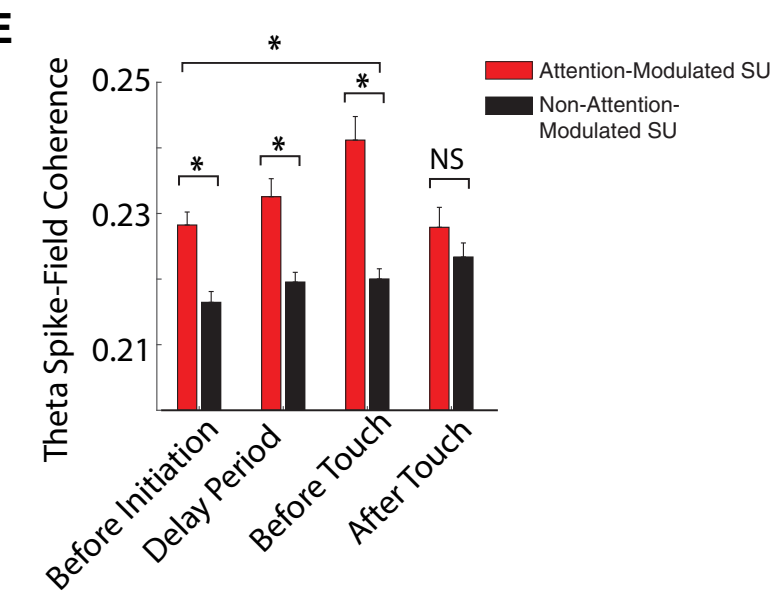

Figure 3. Interaction of spiking activity and the LFP in the $\theta$ band. $\boldsymbol{A}-\boldsymbol{D}$, SFC across sessions and animals for different task epochs for attention-modulated (red) and non-attention modulated units (black). $\boldsymbol{A}$, Before initiation. $\boldsymbol{B}$, Delay period (from initiation to stimulus onset). $\boldsymbol{C}$, Before touch (from stimulus onset to touch response). $\boldsymbol{D}$, After touch. The inset in $\boldsymbol{B}$ shows the STA of the LFP averaged across the attention-modulated (red) and non-attention-modulated units (black), and the shuffle control (green) in the 5-s delay period for all correct trials. The vertical dashed line indicates the spike time. Shaded areas both in the SFC plots and in the STA plot represent SEM. E, SFC of attention-modulated and non-attention-modulated units in $\theta$ band $(4-6 \mathrm{~Hz})$ for different task epochs. $\theta$ Coherence was differentially modulated by task performance. Attention-modulated units showed significantly higher coherence than nonattention-modulated units at all task epochs, except after touch. For attention-modulated units, $\theta$ coherence increased during the delay period compared to before trial initiation and reached the maximum before touch. No change in coherence occurred for non-attention-modulated units during the task; $* p<0.05$; NS indicates $p>0.05$. Error bars represent SEM.

touch) as factors revealed a significant main effect of response type $\left(F_{(1,1035)}=55.8, p<0.001\right)$, main effect of timing $\left(F_{(3,1035)}=4.04, p=0.007\right)$, and significant interaction between these factors $\left(F_{(3,1035)}=4.07, p=0.007\right)$. Post hoc comparison showed that SFC in the $\theta$ frequency band was significantly higher for attention-modulated neurons than for non-modulated neurons during all trial epochs (multiple-comparison with Tukey-Kramer method, before initiation, $p<0.001$, before stimulus onset, $p=0.001$, before touch, $p<0.001$ ), with the exception of the period after the screen touch $(p=0.91)$. While no changes in $\theta$ SFC was observed across task epochs for nonmodulated neurons, $\theta$ SFC was significantly higher before touch than before trial initiation for attention-modulated neurons $(p=0.03)$. These results suggest that neurons whose activity fluctuated with attention selectively synchronized to $\theta$ rhythms based on behavioral demands throughout the task.

\section{$\theta-\gamma$ PAC during attention allocation}

Since $\theta$ rhythms selectively synchronized the spiking activity of attention-modulated neurons, we next examined if the amplitude of $\theta$ rhythms was modulated in a similar way by sustained attention during the 5-CSRTT. Indeed, following trial initiation we observed an increase in $\theta$ power that remained elevated during the sustained attention period (Fig. 4A). $\theta$ Power then peaked $\sim 400 \mathrm{~ms}$ after the presentation of the visual stimulus, before falling to levels lower than before trial initiation. $\theta$ Rhythms have been observed across several cortical and subcortical brain regions, and have been implicated in the functions as diverse as working memory and spatial navigation (Frohlich, 2016). However, these functions of $\theta$ oscillations are underpinned by one unifying mechanism: lower frequency $\theta$ oscillations temporally coordinate higherfrequency $\gamma$ oscillations through PAC. Therefore, to test whether $\theta$ oscillations in LP/pulvinar behaved in a similar 
A

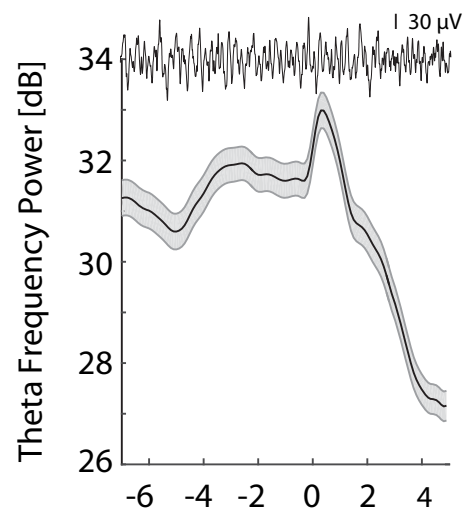

Time from Stimulus Onset [sec]

\section{C}

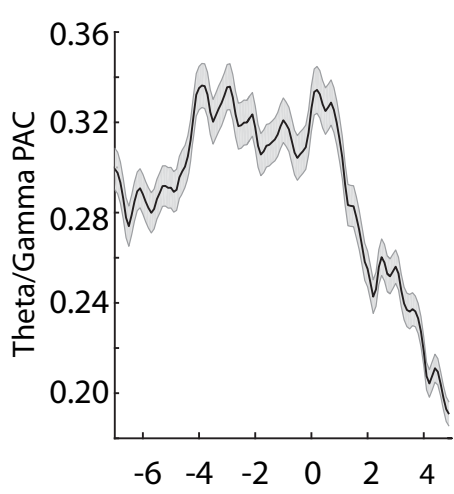

Time from Stimulus Onset [sec]
B

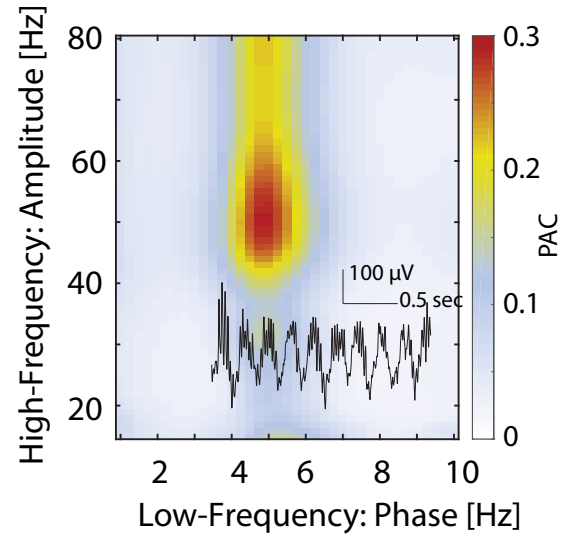

D

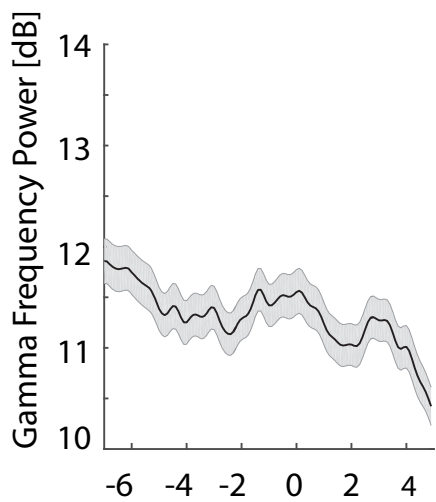

Time from Stimulus Onset [sec]

Figure 4. Task-modulated $\theta / \gamma$ PAC. A, Modulation of $\theta$ power during task performance. $\theta$ Power gradually increased and was maintained during the delay period and peaked between stimulus onset and screen touch. Shaded area represents SEM. An example LFP trace (bandpass in 1-20 Hz) is displayed on the top and shows the $\theta$ oscillation throughout a trial. $\boldsymbol{B}$, Heatmap of PAC during task performance shows selective coupling of $\theta$ and $\gamma$ oscillations. An example LFP trace (bandpass in 1-100 Hz) is superimposed on the heatmap and shows $\gamma-\theta$ oscillation nesting. $\boldsymbol{C}$, Task-dependent modulation of time-resolved $\theta / \gamma$ PAC. $\theta / \gamma$ PAC was elevated throughout the delay period and decreased after screen touch. Shaded area shows SEM. D, Modulation of $\gamma$ power during task performance. $\gamma$ Power gradually decreased during the delay period. Shaded area represents SEM.

way to $\theta$ rhythms observed in other brain regions, we computed PAC between the phase of low-frequency $(<10 \mathrm{~Hz})$ and the amplitude of high-frequency LP/pulvinar LFP signals $(15-80 \mathrm{~Hz})$. This analysis revealed that the amplitude of $\gamma$ oscillations $(30-60 \mathrm{~Hz})$ was temporally coupled to the phase of $\theta$ oscillations $(4-6 \mathrm{~Hz}$, Fig. $4 B$; $\mathrm{PAC}=0.29 \pm 0.01 \mathrm{SEM}$ ), confirming that LP/pulvinar $\theta$ oscillations exhibit similar cross-frequency interaction as $\theta$ observed in other brain regions.

If $\theta / \gamma$ PAC represented an underlying mechanism that LP/pulvinar uses to selectively synchronize cortical inputs and outputs based on behavioral demands, then one would expect the strength of $\theta / \gamma$ PAC to fluctuate across trials of the 5-CSRTT. To test this hypothesis, we computed $\theta / \gamma$ PAC in a temporally resolved manner across the trial. We indeed found task-dependent modulation of $\theta / \gamma$ PAC during performance, where PAC was significantly elevated throughout the sustained attention period, and maintained a high level until screen touch (Fig. 4C; paired $t$ test for PAC values between delay and baseline, $t_{(30)}=$ $4.51, p<0.001, n=31$ recording sessions). We further examined $\gamma$ power across the trials. In contrast to the timecourse of $\theta$ power and $\theta / \gamma$ PAC, we found a significant reduction in $\gamma$ power during the sustained attention period (Fig. $4 D$; paired $t$ test, $t_{(30)}=2.60, p=0.01$ ). Collectively, these results indicate that although $\gamma$ power is reduced during sustained attention, ongoing fluctuations in $\gamma$ amplitude are more tightly locked to the phase of LP/pulvinar $\theta$ oscillations in a behaviorally dependent manner.

\section{Discussion}

We have shown that the LP/pulvinar complex exhibits neuronal dynamics that are modulated during the 5 -CSRTT, in particular during the delay period before onset of the stimulus. We found that oscillatory activity in the $\theta$ frequency binds the neurons that were activated during the delay period. Neuronal firing of these attentionmodulated neurons exhibited a ramp-like increase in their firing rate during the delay period. These results suggest that the LP/pulvinar may play a functional role in sustained attention. 
The pulvinar has only recently become a brain area of wider interest and surprisingly little is known about its function. As a major part of the visual thalamus, the pulvinar appears to play an important role in the control of visual processing and attention (Saalmann and Kastner, 2011). Two recent studies reported somewhat different findings with regards to the role of pulvinar in modulating cortical network dynamics in tasks that required selective spatial attention, which is conceptually distinct from the sustained attention investigated in our study. The work by Saalmann et al. (2012) identified enhanced effective connectivity from pulvinar to cortical areas in the alpha frequency band for attended stimuli. In contrast, work by Zhou et al. (2016a) focused on $\gamma$ band effective connectivity that was directed from cortex to pulvinar, however, they also found an increase in alpha effective connectivity from pulvinar to cortex. Interestingly, inactivation of the pulvinar caused not only a loss of the attentional gain in the cortical stimulus representation but an overall drop in activity below baseline for the unattended stimuli. Thus, these results support a fundamental role of the pulvinar in maintaining and perhaps guiding cortical activity in attention-demanding tasks. Together with our results, these studies suggest that while the pulvinar is involved in multiple domains of attention, the corresponding activity signatures may be distinct.

Other subunits of thalamus also appear to play a role in attention. In the context of selective attention to either visual or auditory stimuli, the medio-dorsal thalamus that forms a recurrent loop with prefrontal cortex plays a key role in amplifying PFC activity that is specific to the stimulus modality used on a given trial (Schmitt et al., 2017). In addition, the nucleus reticularis thalami, which contains inhibitory neurons that provide inhibition to other thalamic areas, is recruited in the same task (Wimmer et al., 2015). Although these studies have investigated other types of attention than the sustained attention probed with the 5-CSRTT they, in agreement with our results, propose a role of the thalamus in allocation of processing resources.

Generators of $\theta$ oscillations have been previously described in the thalamus of animal models (Tsanov et al., 2011) and humans (Sweeney-Reed et al., 2014). $\theta$ Rhythms in the LP/pulvinar of the cat were modulated by the state of vigilance and differed from $\theta$ oscillations recorded from hippocampus (Crighel et al., 1978). In humans, thalamic $\theta$ rhythms, in particular the anterior thalamic nucleus, have been implicated in memory formation (Sweeney-Reed et al., 2015). In addition, human $\theta$ oscillations exhibit PAC with higher-frequency oscillations in the range of $80-150 \mathrm{~Hz}$ (Sweeney-Reed et al., 2017). Despite the differences in the frequency of the amplitudemodulated signal and the thalamic area, the parallel to our findings of $\theta$ oscillations modulating $\gamma$ oscillation is of note. In quite a different context, $\theta$ bursting in thalamus is considered to be a signature of what is called thalamocortical dysrhythmia syndrome (Llinás and Steriade, 2006). In this model, aberrant $\theta$ oscillations (in the form of bursts) emerge due to the deinactivation of the transient, low-threshold calcium current due to deafferentation or other pathology. Together with the fact that thalamic $\theta$ oscillations are associated with decreased vigilance, the question arises why we found an increase in the $\theta$ oscillations in the delay period of the 5-CSRTT. One potential answer derives from the comparison of first-order and higher-order thalamic structures. High-order thalamic structures such as the LP/pulvinar exhibit a substantially larger fraction of rhythmically bursting cells in the awake state (Ramcharan et al., 2005). Thus, rhythmic synchronized activity in higher-order thalamus could serve as a "wake-up call" to cortex due to the enhanced postsynaptic effect of such synchronized thalamic activity and thus support sustained attention

The functional characterization of thalamic networks in ferrets is in its infancy. Little is known about the connectivity of different thalamic nuclei. We recently reported neurochemical subdivision of what we referred to as the LP/pulvinar complex (Yu et al., 2016). By combining tracer techniques and multisite electrophysiology in the anesthetized animal we showed an agreement of structural and functional connectivity between the lateral aspect of the LP/pulvinar complex and visual cortex. However, we can assume that this thalamic area also connects to other, higher-order (visual) areas such as posterior parietal cortex. Thus, we propose that thalamic activity may coordinate cortical activity in the visual areas for successful execution of the 5-CSRTT. Future studies are need to further examine and test this proposed conceptual model. It is worth noting that the nature of extracellular electrophysiology does not allow for the identification of specific cell types within an area. However, we are confident about the anatomic location of the neurons described in this study based on our histologic reconstructions of the electrode tracks and our previous work on the anatomy of the LP/pulvinar complex. An additional source of confusion is the different nomenclature for seemingly similar structures across species, with different naming conventions for carnivores that include the ferret (Jones, 2007). We decided to use the broader term of the LP/pulvinar complex for extrageniculate visual thalamus to avoid dogmatic disputes of researchers across model species (Kaas, 2007). We argue that the structural connectivity (as previously reported by us) is the more relevant information that the specific naming scheme chosen.

To our knowledge, this is the first electrophysiological study of higher-order thalamus in sustained attention using the 5-CSRTT. However, as any scientific study, the work presented here has limitations. First, our findings on the organization of the network activity in the LP/pulvinar complex are correlative in nature and we have not used causal circuit interrogation strategies such as optogenetics. The use of these techniques in larger-brain species such as ferrets and nonhuman primates is still under active development and has remained in its infancy in comparison to the investigations of the mouse brain. We argue that the synthesis of research from different model species with different levels of brain complexity substantially advances the field even if the toolsets vary between them. Nevertheless, the development of targeted causal circuit perturbations in species such as the ferret are of fundamental importance and we recently reported the first 
successful use of optogenetics in the awake behaving ferret (Zhou et al., 2016c). Second, we have not parameterized our tasks for a more detailed dissection of the behavioral components. For example, different lengths of the delay period and presentation of distracting stimuli during the delay period are experimental manipulations that will allow the future testing of hypotheses built on the results from the study presented here. Importantly, introducing competing sensory stimuli would transform the task to one that probes selective attention (Robbins, 2002), which differs from sustained attention. Third, and lastly, we did not investigate how the thalamic signaling modulated corticocortical and cortico-thalamo-cortical interactions. The goal of the current study was to delineate the role of the LP/pulvinar complex in the ferret during sustained attention. Similar investigations with multiple electrode arrays not only in thalamus but also cortical areas that are anatomically connected will be the next step.

In conclusion, our study suggests that the higher-order visual thalamus is engaged during sustained attention and that oscillatory activity in the $\theta$ frequency band organizes the neural firing of the subpopulation modulated by sustained attention. Our findings reinforce the importance of the thalamus in cognitive constructs such as attention that are often studied from a cortico-centric perspective.

\section{References}

Bari A, Dalley JW, Robbins TW (2008) The application of the 5-choice serial reaction time task for the assessment of visual attentional processes and impulse control in rats. Nat Protoc 3:759-767. CrossRef

Barkley RA (1997) Behavioral inhibition, sustained attention, and executive functions: constructing a unifying theory of ADHD. Psychol Bull 121:65-94. CrossRef

Bellgrove MA, Hawi Z, Gill M, Robertson IH (2006) The cognitive genetics of attention deficit hyperactivity disorder (ADHD): sustained attention as a candidate phenotype. Cortex 42:838-845. CrossRef

Bokil H, Purpura K, Schoffelen JM, Thomson D, Mitra P (2007) Comparing spectra and coherences for groups of unequal size. $\mathrm{J}$ Neurosci Methods 159:337-345. CrossRef

Brainard DH (1997) The psychophysics toolbox. Spat Vis 10:433436. Medline

Clark L, Iversen SD, Goodwin GM (2002) Sustained attention deficit in bipolar disorder. Br J Psychiatry 180:313-319. CrossRef

Cohen R, Lohr I, Paul R, Boland R (2001) Impairments of attention and effort among patients with major affective disorders. J Neuropsychiatry Clin Neurosci 13:385-395. CrossRef

Cohen RA, Sparling-Cohen YA, O'Donnell BF (1993) The neuropsychology of attention. New York: Plenum Press.

Crighel E, Kreindler A, Sirian S (1978) Relations between the pulvinarlateralis posterior complex of the thalamus and the hippocampus in wakefulness and sleep in cats. Acta Neurobiol Exp (Wars) 38:167-178.

Day M, Pan JB, Buckley MJ, Cronin E, Hollingsworth PR, Hirst WD, Navarra R, Sullivan JP, Decker MW, Fox GB (2007) Differential effects of ciproxifan and nicotine on impulsivity and attention measures in the 5-choice serial reaction time test. Biochem Pharmacol 73:1123-1134. CrossRef

Frohlich F (2016) Network neuroscience. Boston: Elsevier.

Johnson KA, Robertson IH, Kelly SP, Silk TJ, Barry E, Dáibhis A, Watchorn A, Keavey M, Fitzgerald M, Gallagher L, Gill M, Bellgrove MA (2007) Dissociation in performance of children with ADHD and high-functioning autism on a task of sustained attention. Neuropsychologia 45:2234-2245. CrossRef

Jones EG (2007) The thalamus. Cambridge: Cambridge University Press.

Kaas JH (2007) The thalamus revisited: where do we go from here? Brain 130:2470-2473. CrossRef

Lewin JS, Friedman L, Wu D, Miller DA, Thompson LA, Klein SK, Wise AL, Hedera P, Buckley P, Meltzer H, Friedland RP, Duerk JL (1996) Cortical localization of human sustained attention: detection with functional MR using a visual vigilance paradigm. J Comput Assist Tomogr 20:695-701. CrossRef

Liu SK, Chiu CH, Chang CJ, Hwang TJ, Hwu HG, Chen WJ (2002) Deficits in sustained attention in schizophrenia and affective disorders: stable versus state-dependent markers. Am J Psychiatry 159:975-982. CrossRef Medline

Llinás RR, Steriade M (2006) Bursting of thalamic neurons and states of vigilance. J Neurophysiol 95:3297-3308. CrossRef Medline

Martínez-Arán A, Vieta E, Colom F, Reinares M, Benabarre A, Gastó C, Salamero M (2000) Cognitive dysfunctions in bipolar disorder: evidence of neuropsychological disturbances. Psychother Psychosom 69:2-18. CrossRef

Mirsky AF, Anthony BJ, Duncan CC, Ahearn MB, Kellam SG (1991) Analysis of the elements of attention: a neuropsychological approach. Neuropsychol Rev 2:109-145. CrossRef

Paelecke-Habermann Y, Pohl J, Leplow B (2005) Attention and executive functions in remitted major depression patients. J Affect Disord 89:125-135. CrossRef

Ramcharan EJ, Gnadt JW, Sherman SM (2005) Higher-order thalamic relays burst more than first-order relays. Proc Natl Acad Sci USA 102:12236-12241. CrossRef

Riccio CA, Reynolds CR, Lowe P, Moore JJ (2002) The continuous performance test: a window on the neural substrates for attention? Arch Clin Neuropsychol 17:235-272. CrossRef

Robbins T (2002) The 5-choice serial reaction time task: behavioural pharmacology and functional neurochemistry. Psychopharmacology 163:362-380. CrossRef

Saalmann YB, Kastner S (2011) Cognitive and perceptual functions of the visual thalamus. Neuron 71:209-223.

Saalmann YB, Pinsk MA, Wang L, Li X, Kastner S (2012) The pulvinar regulates information transmission between cortical areas based on attention demands. Science 337:753-756. CrossRef

Sarter M, Givens B, Bruno JP (2001) The cognitive neuroscience of sustained attention: where top-down meets bottom-up. Brain Res Rev 35:146-160. CrossRef

Schmitt LI, Wimmer RD, Nakajima M, Happ M, Mofakham S, Halassa MM (2017) Thalamic amplification of cortical connectivity sustains attentional control. Nature 545:219-223.

Sellers KK, Yu C, Zhou ZC, Stitt I, Li Y, Radtke-Schuller S, Alagapan S, Fröhlich $F$ (2016) Oscillatory dynamics in the frontoparietal attention network during sustained attention in the ferret. Cell Rep 16:2864-2874. CrossRef

Shoham S, Fellows MR, Normann RA (2003) Robust, automatic spike sorting using mixtures of multivariate t-distributions. J Neurosci Methods 127:111-122. CrossRef

Stitt I, Zhou Z, Radtke-Schuller CS, Frohlich F (2017) Arousal dependent modulation of thalamo-cortical functional interaction. bioRxiv

Sweeney-Reed CM, Zaehle T, Voges J, Schmitt FC, Buentjen L, Kopitzki K, Esslinger C, Hinrichs H, Heinze H-J, Knight RT, Richardson-Klavehn A (2014) Corticothalamic phase synchrony and cross-frequency coupling predict human memory formation. Elife 3:e05352. CrossRef

Sweeney-Reed CM, Zaehle T, Voges J, Schmitt FC, Buentjen L, Kopitzki K, Hinrichs H, Heinze H-J, Rugg MD, Knight RT, Richardson-Klavehn A (2015) Thalamic theta phase alignment predicts human memory formation and anterior thalamic crossfrequency coupling. Elife 4:e07578. CrossRef

Sweeney-Reed CM, Zaehle T, Voges J, Schmitt FC, Buentjen L, Borchardt V, Walter M, Hinrichs H, Heinze H-J, Rugg MD, Knight RT (2017) Anterior thalamic high frequency band activity is coupled 
with theta oscillations at rest. Front Hum Neurosci 11:358. CrossRef

Tsanov M, Chah E, Wright N, Vann SD, Reilly R, Erichsen JT, Aggleton JP, O'Mara SM (2011) Oscillatory entrainment of thalamic neurons by theta rhythm in freely moving rats. J Neurophysiol 105:4-17. CrossRef

Wimmer RD, Schmitt LI, Davidson TJ, Nakajima M, Deisseroth K, Halassa MM (2015) Thalamic control of sensory selection in divided attention. Nature 526:705-709. CrossRef

Yu CX, Sellers KK, Radtke-Schuller S, Lu JH, Xing L, Ghukasyan V, Li YH, Shih YYI, Murrow R, Fröhlich F (2016) Structural and func- tional connectivity between the lateral posterior-pulvinar complex and primary visual cortex in the ferret. Eur J Neurosci 43:230-244. CrossRef

Zhou H, Schafer RJ, Desimone R (2016a) Pulvinar-cortex interactions in vision and attention. Neuron 89:209-220.

Zhou ZC, Salzwedel AP, Radtke-Schuller S, Li Y, Sellers KK, Gilmore JH, Shih YI, Fröhlich F, Gao W (2016b) Resting state network topology of the ferret brain. Neuroimage 143:70-81. CrossRef

Zhou ZC, Yu C, Sellers KK, Fröhlich F (2016c) Dorso-lateral frontal cortex of the ferret encodes perceptual difficulty during visual discrimination. Sci Rep 6:23568. CrossRef 